\title{
Multifocal electroretinography in patients with Stargardt's macular dystrophy
}

\author{
U Kretschmann, M W Seeliger, K Ruether, T Usui, E Apfelstedt-Sylla, E Zrenner
}

\begin{abstract}
Aims-To describe the topography of multifocal electroretinograms (ERGs) and to explore its diagnostic value in patients with Stargardt's macular dystrophy (SMD).

Methods-51 patients with SMD were examined by means of the $\mathrm{m}$-sequence technique to characterise the topography of electroretinographic responses in the central visual field. The results were compared with data from 30 normal volunteers.

Results-In 49 of 51 patients with SMD, macular electroretinographic activity was markedly diminished or non-detectable. Towards more peripheral areas, ERG responses of the SMD patients approached those of normals. Implicit times were not markedly delayed at any eccentricity.

Conclusion-In contrast with Ganzfeld electroretinography, multifocal electroretinography is useful to detect foveal dysfunction in SMD. Areas of dysfunction were found to be usually larger than expected from psychophysical measurements and morphological alteration. In early stages of the disease it was possible to detect foveal dysfunction, even in patients lacking morphological fundus changes and with good visual acuity.

(Br f Ophthalmol 1998;82:267-275)
\end{abstract}

In 1909 Stargardt described a unique macular dystrophy characterised by visual loss in the first two decades of life and an atrophic lesion of the macula. ${ }^{1}$ The term fundus flavimaculatus (FF) in conjunction with Stargardt's disease was first used by Franceschetti in $1963^{2}$ and denotes a retinal dystrophy characterised by yellow flecks, found in the distal retina at the posterior pole of the eye. Based on later studies both phenotypes are regarded as reflecting different expression of the same hereditary disease. $^{3-6}$ The main and in most cases the only complaint is a loss of central vision.?

In histological studies the primary site of the disease was found in the RPE followed by an early degeneration of the photoreceptors. ${ }^{8-11}$ However, electroretinography (ERG) and electro-oculography (EOG) did not turn out to be valuable diagnostic tools because functional loss is initially restricted to small circumscribed areas of the retina. Therefore, reduced Ganzfeld ERG amplitudes are only found in advanced stages of the disease, making up 16\% of 50 cases observed by Noble and Carr. ${ }^{5}$ Fishman reported that the Arden ratio of the EOG is pathological in a high percentage of the stages III and IV where diffuse changes of the RPE are already visible by ophthalmoscopy and fluorescein angiography. ${ }^{4}$ Noble and Carr found that although macular changes are common $(94 \%)$, there are patients in early stages in which morphological changes are very subtle, but visual acuity is already remarkably reduced. ${ }^{5}$ Differential diagnoses in such cases include cone dystrophies, functional visual loss, and diseases of the higher visual pathways.

The multifocal ERG technique of Sutter and $\operatorname{Tran}^{12}$ allows the electroretinographic activity in the central visual field to be mapped. The stimulation of the different areas during examination occurs simultaneously in a pseudo random manner and each focal ERG is calculated from the raw data by a cross correlation technique which extracts linear and non-linear components. The linear component, the so called first order kernel, has been shown to provide information from the outer retinal layers. Hood et al found a close relation between the first order kernel response of the multifocal ERG and the Ganzfeld ERG in parametric studies. ${ }^{13}$ The technique is quite novel with respect to other techniques of focal electroretinography - for example, those based on flicker ${ }^{14}{ }^{15}$ or flash stimulation. ${ }^{16}$ Retest variability of multifocal ERG was found comparable with that of Ganzfeld ERG. ${ }^{17}$

First studies in individual patients have shown that central cone dysfunction can be detected by multifocal electroretinography. ${ }^{18-20}$ In the present study, a larger number of patients with Stargardt's macular dystrophy/ fundus flavimaculatus were examined to determine the sensitivity of the method. Besides numerous cases in advanced stages of the disease, patients in early stages with good visual acuity, without marked fundus alterations, or both are also presented.

\section{Subjects and methods}

\section{SUBJECTS}

Fifty one patients with Stargardt's macular dystrophy, some of whom also showed some degree of fundus flavimaculatus were included in this study. Informed consent was obtained from patients and controls after description of the procedures. Tenets of the declaration of Helsinki were followed and institutional human experimentation committee approval obtained. The diagnosis was based on history, symmetric bilateral involvement, the typical alterations of the pigment epithelium layer, assessed by fluorescence angiography if necessary, by visual field, and Ganzfeld electroretinography according to the ISCEV standard. ${ }^{21}$ 
Ages ranged from 7 to 68 years, median 29 years; 27 were male, 24 female. The visual acuity ranged from 0.02 to 1.5 . Forty one eyes had a visual acuity of 0.2 and lower, 28 eyes of 0.6 and higher, and 32 between these values. In all patients central or paracentral scotomata were found. Autosomal recessive inheritance was suggested in eight patients from seven pedigrees. Three patients from an autosomal dominant pedigree were included in the study. In an 8 year old girl, a multifocal ERG could only be obtained from one eye, so that data from 101 eyes were analysed.

Thirty normal volunteers were examined with the multifocal ERG to serve as a control group. With appropriate correction they had a full visual acuity. Refraction errors were in the range of plus or minus 6.0 dioptres, and there was no history of eye diseases and no serious general diseases. Their age ranged from 22 to 58 years, median 31 years.

STIMULATION AND RECORDING PROCEDURES OF THE MULTIFOCAL ERG

The stimulus was presented on a monitor with a frame rate of $75 \mathrm{~Hz}$ at a distance of $28 \mathrm{~cm}$ from the subject's eye. It consisted of 61 hexagons within a 30 degree visual field (Fig 1A). The radius of the central hexagon was 2 degrees. The hexagon areas increased with eccentricity, so that the most eccentric hexa- gons were 4.7 times larger than the central one. Each element changed between black and white $(93 \%$ contrast) following a binary $\mathrm{m}$-sequence independently from other elements. A new stimulus picture was presented every $13 \mathrm{~ms}$. The mean luminance was 51.8 $\mathrm{cd} / \mathrm{m}^{2}$. A red central fixation point of $2 \mathrm{~mm}$ diameter was used. For stimulation and data analysis the VERIS system for the Macintosh Quadra provided by Dr Sutter, EDI, San Francisco was used.

Refractive errors were corrected and the pupils dilated with tropicamide $(0.5 \%)$ and phenylephrine (5\%). The ERG responses were recorded by means of DTL fibre electrodes (UniMed Electrode Supplies, England) which were positioned on the conjunctiva directly beneath the cornea and attached with its two ends at the lateral and nasal canthus. The reference and skin electrodes (gold cup electrodes) were attached to the ipsilateral temple and forehead, respectively.

The electroretinograms were amplified $(\times 200000)$ and band pass filtered (10-100 $\mathrm{Hz}$ ) using a Grass amplifier (model 12, Quincy, USA).

The duration of the recording session was about 5-7 minutes, which included 20 recording segments of 10 seconds each, during which the subjects were not allowed to blink or move. Fixation was controlled by direct observation
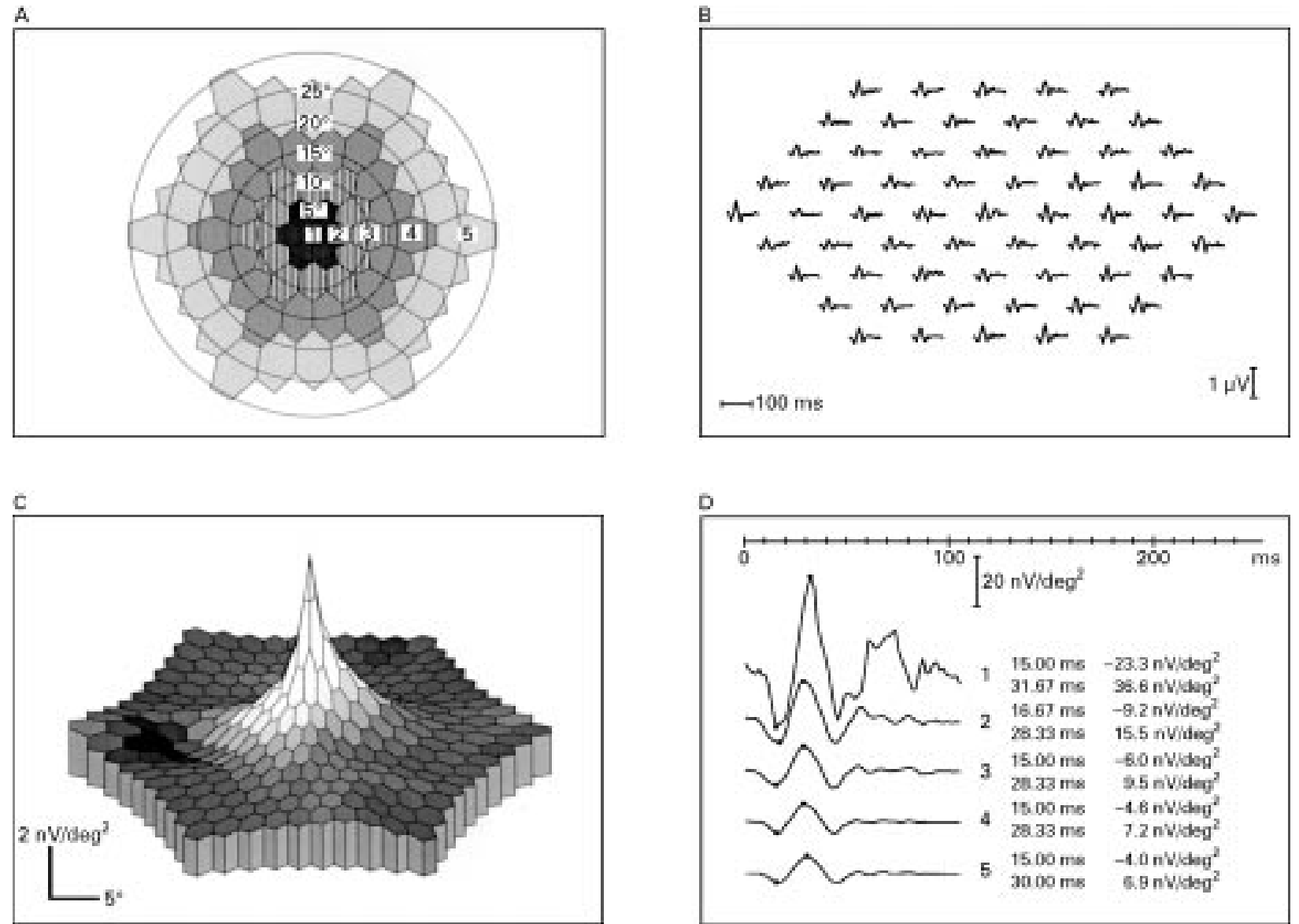

Figure 1 (A) Geometry of the stimulus. The 61 hexagonal elements were grouped in five rings. (B) Trace array of 61 first order kernel ERG waves from the left eye of a normal volunteer. (C) Response density of the same eye as in (B) calculated as scalar product plotted. The central peak corresponds to the macular area and the minimum on the left to the area of the optic nerve head (blind spot). (D) Averaged ERGs of the five groups indicated in (A) from the same eye as in $(B)$ and $(C)$. The implicit time was measured from stimulus onset to the first positive peak. 

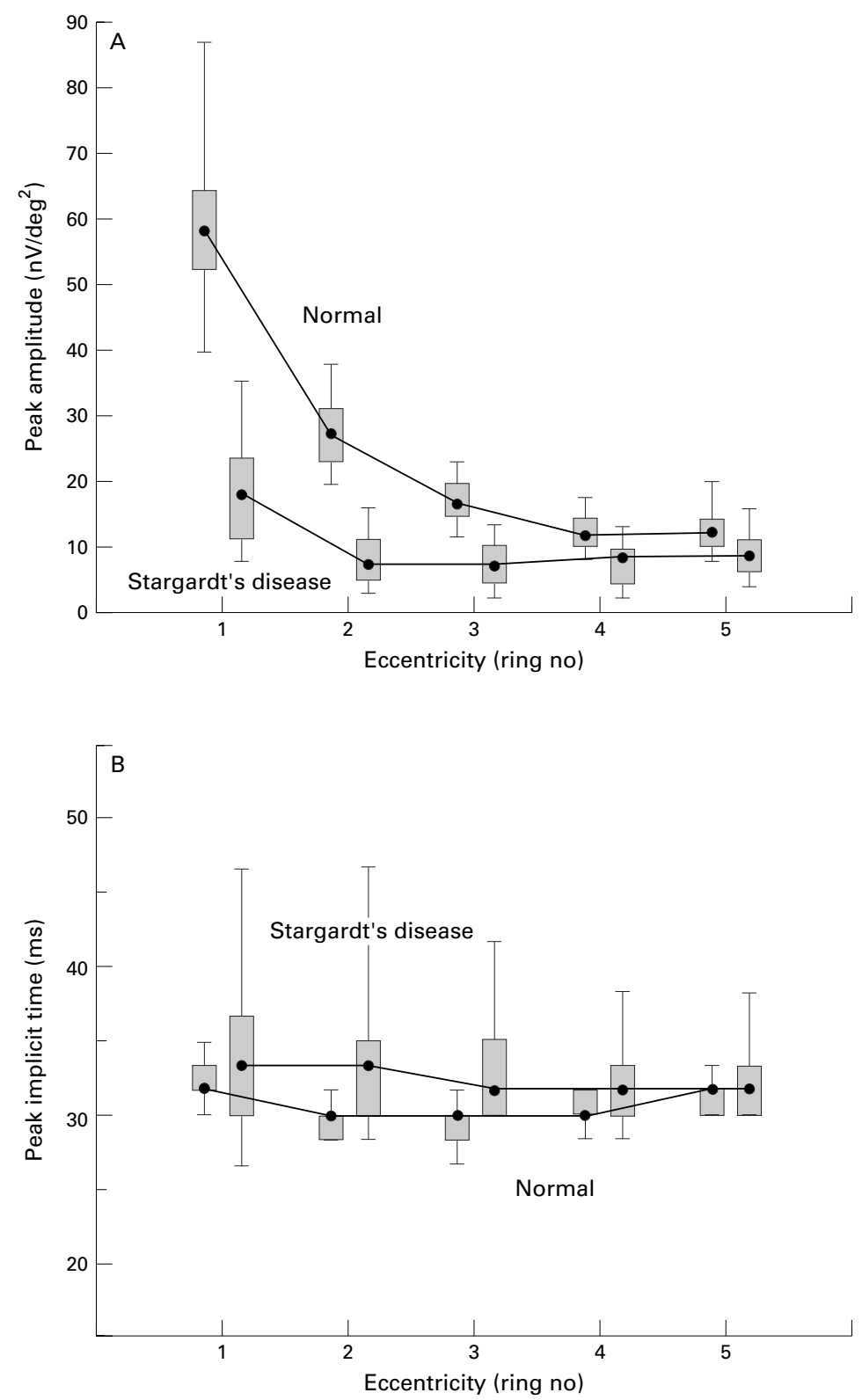

Figure 2 Response density $(A)$ and implicit time (B) in normal controls and patients with SMD versus eccentricity. Numbers on the abscissa indicate eccentricity groups (see Fig $1 A$ ). The values were calculated only from data obtained in left eyes. The black dots indicate the median, the shaded box the $25 \%$ and $75 \%$ quartiles, the end of the error bars the $5 \%$ and $95 \%$ quantiles. grouped in concentric rings as shown in Figure 1A. Averaging within these groups yields five sum responses as shown in Figure 1D, trace 1 representing the innermost hexagon (ring 1). The implicit time was measured in each group from the onset of the stimulus to the first positive peak of the response. A peak to peak amplitude was measured from the first negative peak to the first positive peak. This amplitude was divided by the area from which the respective amplitude was evoked, resulting in a measure of response density in $\mathrm{nV} / \mathrm{deg}^{2}$, as shown in Figure 1D for a normal volunteer.

Five per cent, $25 \%, 50 \%, 75 \%$, and $95 \%$ quantiles were calculated from amplitude and implicit time data of SMD patients and normals. Differences between groups were analysed using the non-parametric MannWhitney test. Differences were considered significant at a level of $\mathrm{p}<0.05$. Trial to trial variability was tested in 10 normals with two consecutive measurements. There was an average difference of amplitudes in the fovea of $8.8 \%$ (range $0.4 \%-20.6 \%$ ) and in the periphery (ring 5) of $6.7 \%$ (range $1.1 \%-16.3 \%$ ).

\section{Results}

In Figure 2, response densities (A) and implicit times (B) in 30 normals and 51 SMD patients are shown for comparison. In the macula-that is, ring 1 and 2 up to $7^{\circ}$ eccentricity, the response density of the SMD patients was lower than that of the normal controls without an overlap of the 5-95\% intervals (Fig 2A). There were two SMD patients without marked macular dysfunction, a 26 year old man with a visual acuity of $0.8 / 0.6$, and a woman aged 22 with a visual acuity of $0.5 / 0.6$ who showed only a mildly subnormal foveal ERG (group 1 up to $2^{\circ}$ eccentricity) in the left eye. A male patient aged 43 with a visual acuity of 1.5 and a diagnosis of atypical FF showed large foveal responses, but markedly decreased ERG activity from eccentricity groups $2-5$. In all other patients the responses in all seven macular elements (eccentricity groups 1 and 2) were decreased. In group 3 (5-13 ${ }^{\circ}$ eccentricities), the response density was lower in the affected group, but there was some overlap between both groups. In Figure 3, the response densities are shown for each individual eye. There is only a small number of eyes (seven) with subnormal foveal ERG but normal ERGs in group 3, indicating that the functional defect is not limited to the macula in the majority of cases. The response density in the most peripheral eccentricity groups 4 and 5 (10$31^{\circ}$ ) was only slightly smaller than that measured in the control groups (Fig 2A); however, the differences in all five eccentricities were statistically significant. Looking at all 101 eyes individually, a relatively high number of 32 eyes was found to have decreased amplitudes also in the most peripheral areas (Fig 3B). It should be noted though that the signals from peripheral areas were clearly discernible from noise in all patients, while the signals in the macular region were frequently within noise level. 

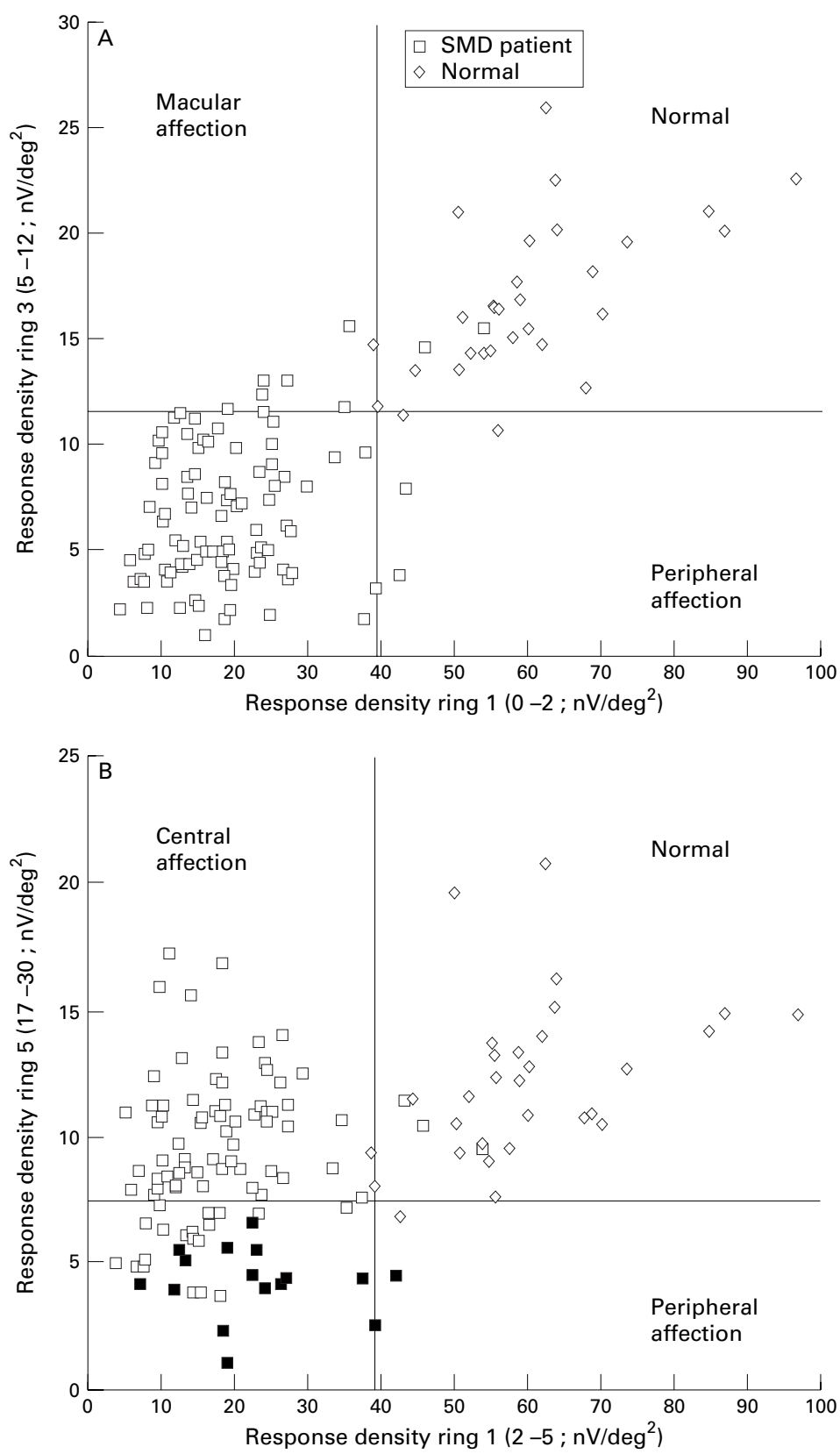

Figure 3 Comparison of response densities of 101 eyes of SMD patients and 30 normal eyes in different topographic regions. Lines indicate the border of $95 \%$ percentiles of the normals. In $(A)$ response densities of ring $3\left(5^{\circ}-12^{\circ}\right)$ are plotted against response densities of ring 1 (fovea). In (B) response densities of ring $5\left(17^{\circ}-30^{\circ}\right)$ are plotted against response densities of ring 1 (fovea). Filled squares indicate eyes with pathological photopic Ganzfeld ERGs.

In 45 of the 51 SMD patients, a photopic Ganzfeld ERG was obtained. Pathological responses to white flash stimulation were found in eight cases $(17.8 \%)$. All patients from the latter group showed delays in b-wave implicit time while only three of them also showed reduced amplitudes. In all patients who had pathological Ganzfeld ERGs, abnormalities in multifocal ERG were found at all eccentricity groups up to $30^{\circ}$ of the visual field. These eyes are indicated by filled squares in Figure 3B. These patients could be differentiated from patients with cone dystrophies by the clinical picture and by the fact that the cone Ganzfeld ERG was only moderately subnormal.
Implicit times were only slightly higher than in normal controls; however, these differences were significant in eccentricity groups $2-5$ field (Fig 2B). In the central areas (groups 1-3) it was often difficult to determine a clear peak because of marked alterations in ERG wave form resulting in a higher variability of implicit times. In eccentricity groups 4 and 5 with clearly detectable signals, a subgroup of six patients with both decreased response densities and increased implicit times was discernible. Five of these six patients $(83.3 \%)$ also exhibited a pathological photopic Ganzfeld flash ERG indicating a more widespread cone dysfunction.

After this more general analysis, we now present some special problems.

EARLY STAGES OF THE DISEASE

A major diagnostic problem is the early stages of SMD. Seven such young patients aged 7 to 12 were included in this study. The macular electroretinographic activity was markedly diminished in all of them.

Case 1, an example of an early stage of the disease, is presented in Figure 4. The 12 year old male patient had noticed a reduction of visual acuity since age 8 , combined with an increase in glare sensitivity. Best corrected visual acuity was 0.4 in both eyes. Anterior segments were unremarkable. Ophthalmoscopy revealed a regular optic nerve head, parafoveolar defects of the pigment epithelium with granular appearance, and some peripheral yellow flecks most prominent in the region of the major vessels (Fig 4A). Static perimetry (Tübingen automatic perimeter) revealed a small central scotoma in both eyes (Fig 4B) The desaturated panel D15 test showed some errors along the tritan axis. In Ganzfeld ERG, normal scotopic and photopic responses were recorded.

The multifocal ERG exhibited markedly diminished electroretinographic responses in a relatively small circumscribed area surrounded by normal focal ERGs (Fig 4C, D). While the young patient had some difficulties in keeping fixation during static perimetry, there were no fixation problems during the 5 minutes of recording of multifocal ERG as demonstrated by the locally reduced electrical responses in the areas of the blind spots.

Additionally, there have been five patients with only minor changes in fundus morphology such as case 2 , a 20 year old male patient complaining of loss of visual acuity since age 16. Corrected visual acuity was 0.1 in both eyes. IOP and anterior segments were normal. In the macular region, tiny defects of the pigment epithelium and tapetoid reflexes were visible (Fig 5A). Static perimetry (Tübingen automatic perimeter) revealed a relative central scotoma of $5^{\circ}$ in diameter (Fig 5B). Defects of colour vision along the deutan axis were found in the panel D15 test while Ganzfeld ERGs and EOG revealed no abnormal findings.

In the multifocal ERG the macular responses were diminished to noise level and surrounded by clear responses of normal amplitude (Fig 5C, D). 
A

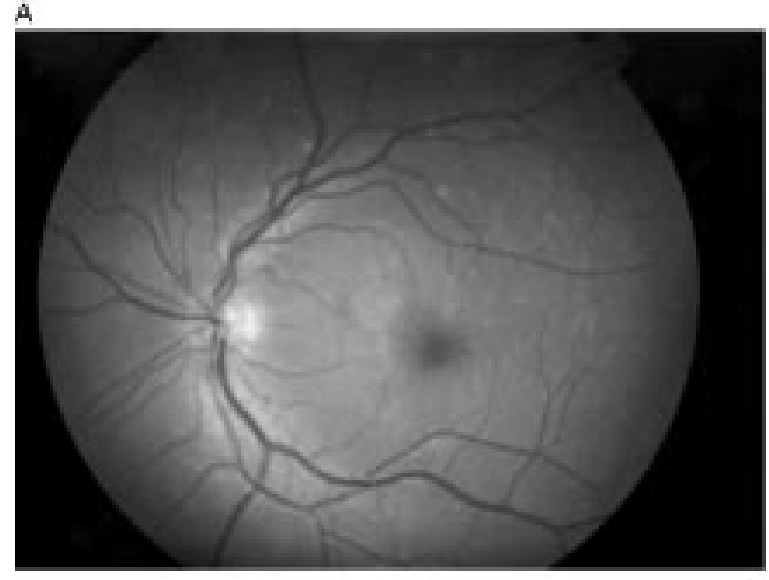

c

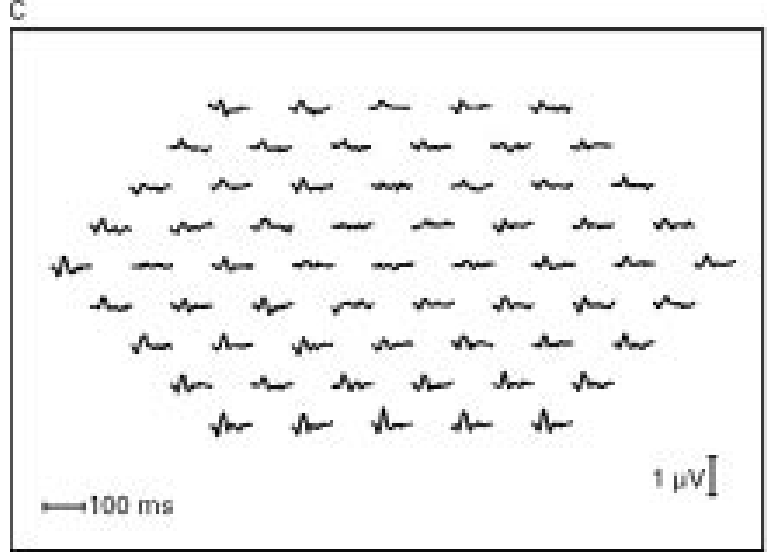

B

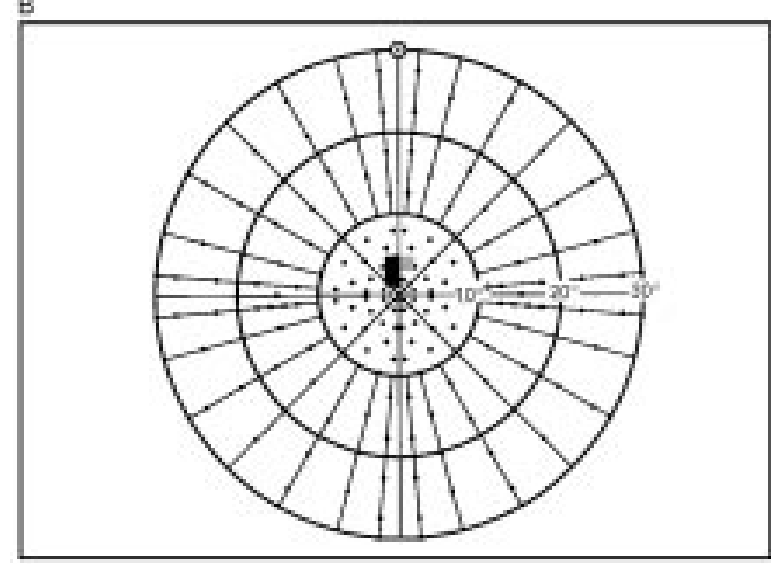

D

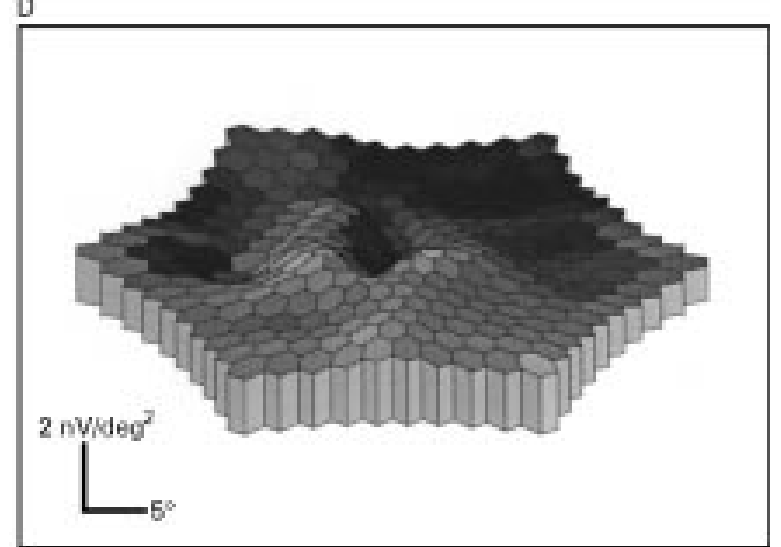

Figure 4 Case 1 left eye. (A) Fundus photograph. (B) Visual field recorded with Tübingen static perimetry, background luminance 10 cd/m ${ }^{2}$, maximal luminance $1000 \mathrm{~cd} / \mathrm{m}^{2}$, stimulus size 10'. Small squares indicate normal light sensitivity, large open squares relative defects of 5-10 dB, large shaded squares relative defects $>10 \mathrm{~dB}$, large solid squares absolute defects. (C) Trace array of 61 focal ERGs. (D) Plot of response density.

RELATION TO VISUAL ACUITY

In 25 out of $28(89.3 \%)$ SMD eyes with a visual acuity of better than 0.6 the foveal response density was below the normal 5\% percentile. Owing to this high sensitivity even in cases with good visual acuity, only a weak correlation could be found between the foveal response density and visual acuity $(r=0.05)$ and central differential light sensitivity (measured during perimetry as increment threshold; $r=0.062$ ).

This is illustrated in case 3 (Fig 6), a 25 year old female with a best corrected visual acuity of 1.0 (right eye) and 0.8 (left eye). Anterior segments and IOP were normal. Macular biomicroscopy revealed an atrophy of the pigment epithelium and yellow flecks which were more pronounced in the right than the left eye (Fig $6 \mathrm{~B}, \mathrm{C})$. Fluorescein angiography showed a dark choroid in both eyes typical of Stargardt's disease. In static perimetry (Tübingen automatic perimeter) a slight decrease of central light sensitivity was observed in the left eye (25 $\mathrm{dB})$ compared with the right $(31 \mathrm{~dB})$. In the right eye, a ring of decreased light sensitivity around the fovea was observed in perimetry, while in the left eye only four shallow relative defects were detected in the parafoveal region (Fig 6C, D). The Ganzfeld ERGs were normal both under scotopic and photopic conditions.
The focal ERG responses from the central region of the left eye were diminished relative to the peripheral responses (Fig 6E). The response density values were below the normal range. The response density distribution showed a flat topography with a small central peak (Fig 6G). The responses of the right eye were even smaller than those of the left (Fig $6 \mathrm{~F})$. There was no foveal signal visible and therefore no peak in the three dimensional topography (Fig 6H). The central response of the multifocal ERG reflects the function of the fovea (up to $2^{\circ}$ eccentricity) better than Snellen acuity or the central light sensitivity.

\section{Discussion}

The multifocal ERG based on the m-sequence stimulation approach ${ }^{22}{ }^{23}$ is capable of mapping the central visual field functionally by means of local electroretinographic responses. As shown by Sutter and $\operatorname{Tran}^{12}$ using recordings with higher spatial resolution and longer recording time, the response density decreases markedly from the foveal area towards the periphery. This response distribution resembles the anatomical distribution of cones $^{24}$ as well as the electroretinographic findings obtained with focal flicker stimulation by Maxwellian view. ${ }^{25}$ A few reports have shown that central cone dysfunction can be detected by multifocal ERG in retinal diseases. ${ }^{18-20}$ 
A

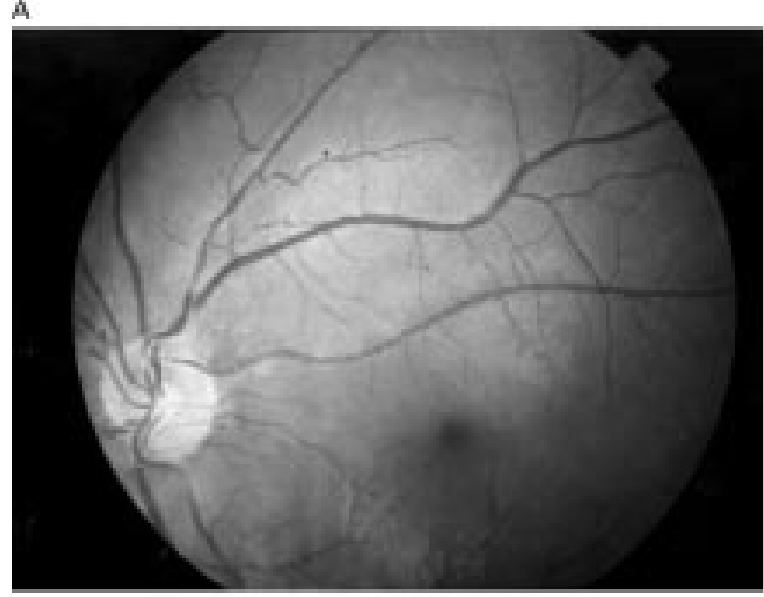

c.

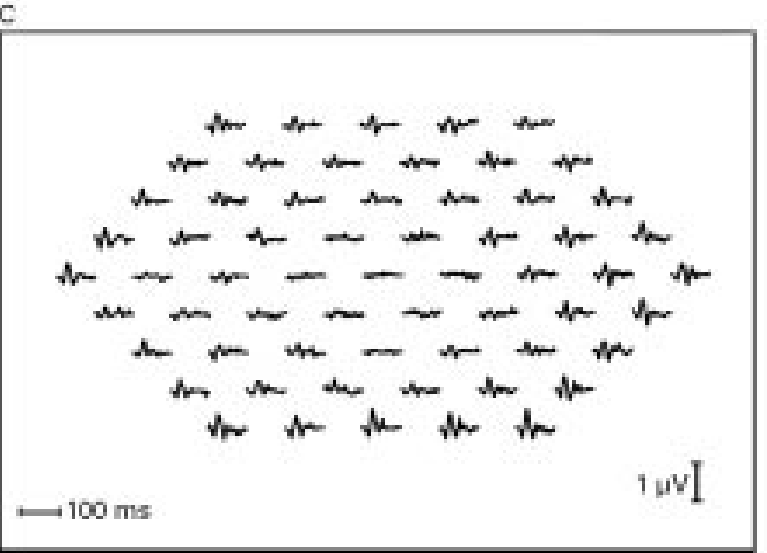

B

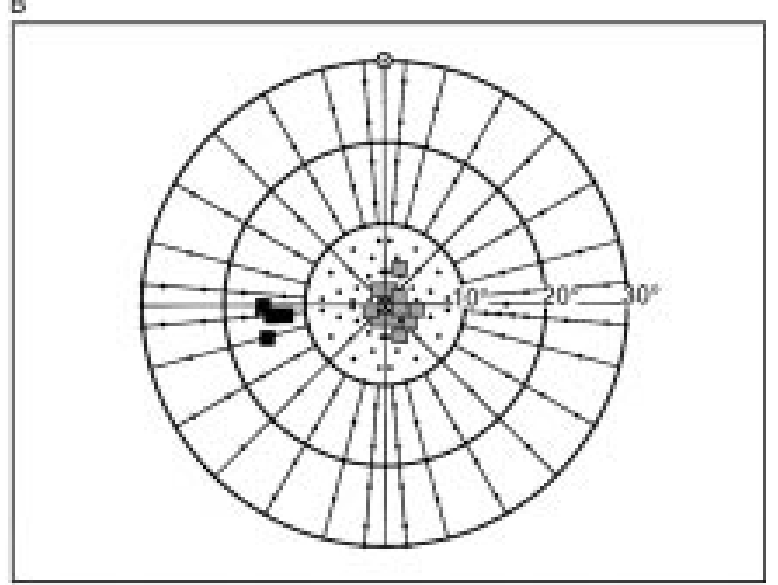

D

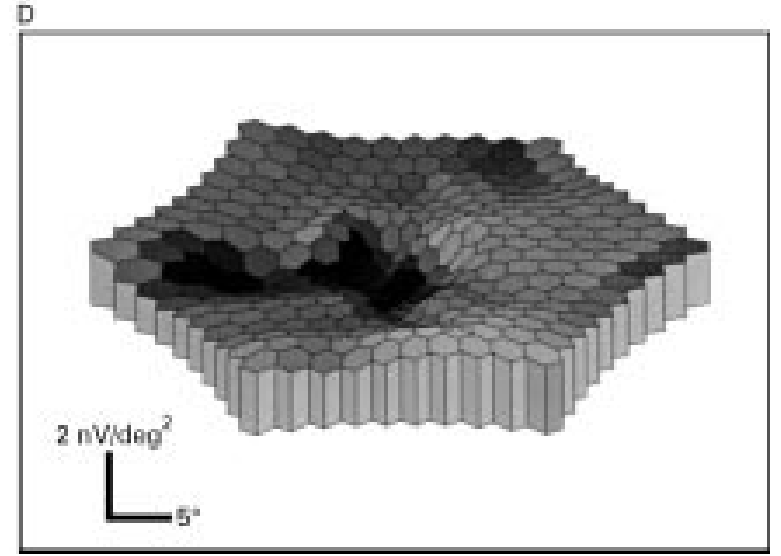

Figure 5 Case 2 left eye. (A) Fundus photograph. (B) Visual field recorded with Tübingen static perimetry. (C) Trace array of 61 focal ERGs. (D) Plot of response density.

Reduced foveal activity has also been measured by other techniques employing macular flicker stimulation ${ }^{141526}$ and pattern electroretinography. ${ }^{27}$ However, these reports included only a small number of patients with SMD.

In this study, we have detected central cone dysfunction in almost all patients with SMD (49/51;96.1\%). Multifocal ERG was found to be particularly helpful in patients with only slight fundus changes and in young patients where the diagnosis is sometimes difficult to establish. Furthermore, in 25 out of 28 eyes with a visual acuity of 0.6 or better, the multifocal ERG indicated regional dysfunctions of the cones.

In addition to the methods of foveal electroretinography, multifocal ERG provides an estimate of the extension of central cone dysfunction. Although SMD is addressed as a juvenile maculopathy, in most of our patients cone dysfunction was not restricted to the macula. On the basis of the response densities at different retinal eccentricities and considering the results obtained in photopic Ganzfeld ERG, we suggest a functional staging of patients with SMD (Table 1). In seven eyes, the defect in multifocal ERG was restricted to the macula. The largest group of eyes $(n=56)$ showed additional abnormalities at eccentricities between 5-12 . Nevertheless, 24 eyes showed a clear decrease of response ampli- tudes in the entire test field. If a more peripheral ring or Ganzfeld ERG was found abnormal with a normal response in a more central region, the case was classified as atypical as in one patient with fundus flavimaculatus. Apart from this patient, there was no correlation of the degree of yellow flecks and the size of the central defect in multifocal electroretinography.

It should be noted that the entire test field used for multifocal ERG recordings in this study covers about $35 \%$ of the entire cone population. It is therefore not surprising that the results in Ganzfeld and multifocal ERG were not strongly correlated. Although the sensitivity of Ganzfeld ERG in SMD was found to be poor $(17.8 \%)$ in our patients, it helps in differentiating progressed stages of the disease, especially if functional defects are present beyond $30^{\circ}$ borders as shown in Table 1.

Although implicit times in SMD and the control group differed significantly in the extrafoveal areas, only 13 eyes of 101 exhibited values outside the confidence interval of the peripheral ring. Most of the patients with abnormal timing in the periphery also had an abnormal photopic Ganzfeld ERG, indicating a more diffuse affection of the cone system. This underlines that implicit times in the multifocal ERG were not as much delayed as found in retinitis pigmentosa, incomplete 
A

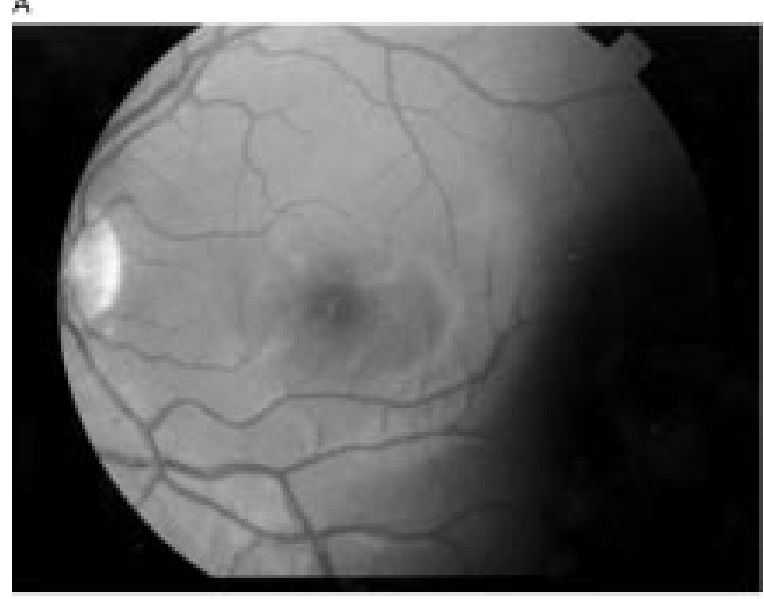

C

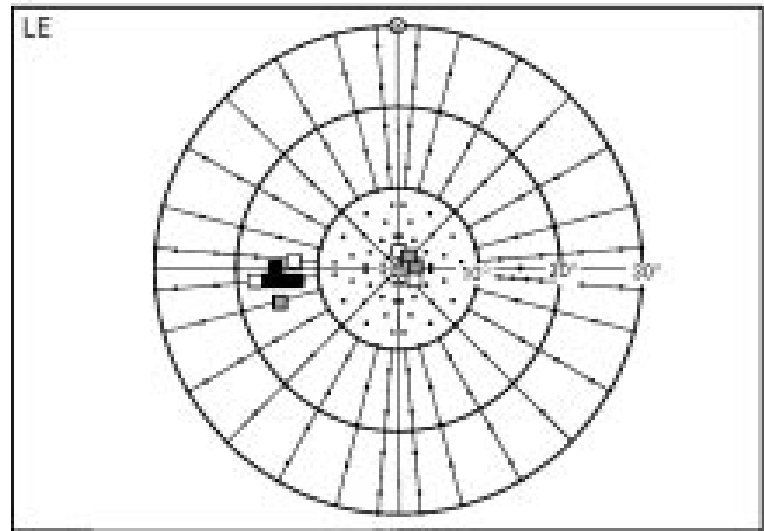

B

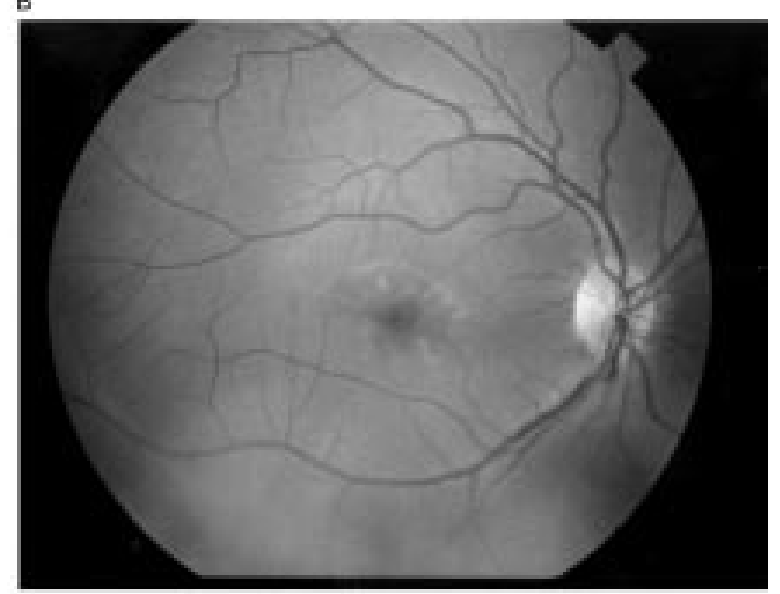

D

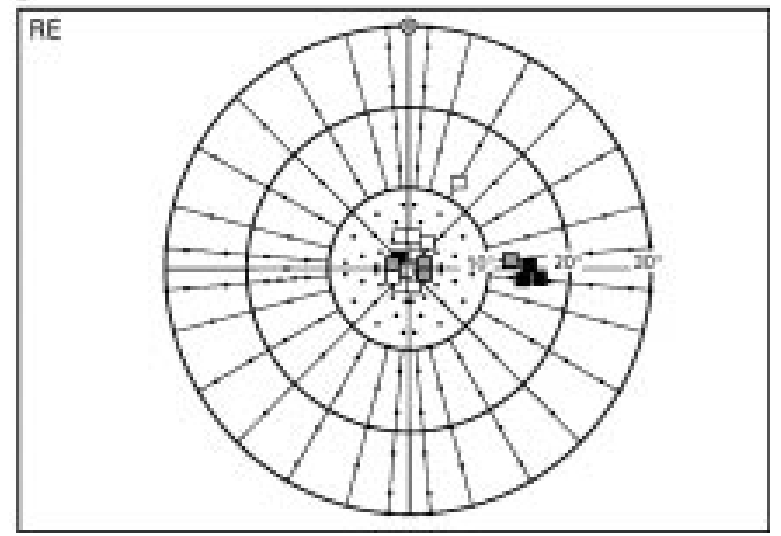

Figure 6 Case 3 both eyes. (A) Fundus photograph (left eye). (B) Fundus photograph (right eye). (C) Visual field recorded with Tübingen static perimetry (left eye). (D) Visual field recorded with Tübingen static perimetry (right eye).

congenital stationary night blindness, and cone dystrophy. ${ }^{28-30}$

The differential diagnosis of SMD with or without fundus flavimaculatus includes all "flecked retina syndromes". ${ }^{631}$ The most common are dominant drusen, fundus albipunctatus, Biettis crystalline retinopathy, and pattern dystrophy. The differential diagnosis of flecked retina diseases is usually based on biomicroscopic observation, but functional testing can also add some information. In a case of Biettis crystalline retinopathy we have found an activity pattern in multifocal $\mathrm{ERG}^{32}$ that would be described as atypical in this

Table 1 Electrophysiological staging using the results of multifocal ERG and photopic Ganzfeld ERG

\begin{tabular}{lll}
\hline Classes & Description & No of eyes \\
\hline I & $\begin{array}{l}\text { Subnormal multifocal ERGs only in } \\
\text { groups } 1 \text { and } 2\left(0^{\circ}-7^{\circ}\right)\end{array}$ & 7 \\
II & $\begin{array}{l}\text { Subnormal multifocal ERGs in } \\
\text { groups } 1-3\left(0^{\circ}-12^{\circ}\right)\end{array}$ & 56 \\
III & $\begin{array}{l}\text { Subnormal ERGs in the entire test } \\
\text { field }\left(0^{\circ}-30^{\circ}\right), \text { but normal Ganzfeld }\end{array}$ & 10 \\
IV & $\begin{array}{l}\text { ERG } \\
\text { Subnormal ERGs in the entire test } \\
\text { field }\left(0^{\circ}-30^{\circ}\right) \text { plus pathological }\end{array}$ & 14 \\
Vanzfeld ERG & $\begin{array}{l}\text { Atypical forms } \\
\text { No defect detectable using the } \\
\text { multifocal ERG }\end{array}$ & 2 \\
\end{tabular}

Ninety three eyes of 47 patients were analysed. (Eight eyes of four patients with subnormal results in the entire test field in multifocal ERG have not been assessed by photopic Ganzfeld ERG. These patients were omitted in the analysis.) paper. Although crystals were present in the fovea, the response of the central element was normal with pathological responses in paramacular areas.

Electrophysiological testing is even more important in cases where ophthalmological findings are sparse or inconclusive. In cone dystrophies, the overall reduction of the multifocal ERG responses is much more pronounced than in SMD, a finding that is already observable in Ganzfeld ERG (30 Hz flicker and photopic flash).

Another differential diagnostic problem is early stages of SMD with subtle morphological changes. As stated by Noble and Carr such patients might be misdiagnosed to have hysteria, malingering, or brain diseases. ${ }^{5}$ Multifocal ERG can elucidate the function of macular cones and lead to a correct diagnosis in such cases.

Multifocal ERG can be useful for the diagnosis and differential diagnosis of SMD and help to describe the topography of cone activity loss more precisely. One of the surprising findings is that functional defects in Stargardt's disease are often not limited to the fovea and parafovea, but commonly affect the retina more than expected by visual field measurements and ophthalmoscopy.

Recently, a defect of the ABCR gene which is expressed in rod photoreceptors was found in two families with autosomal recessive 
274

Kretschmann, Seeliger, Ruether, et al
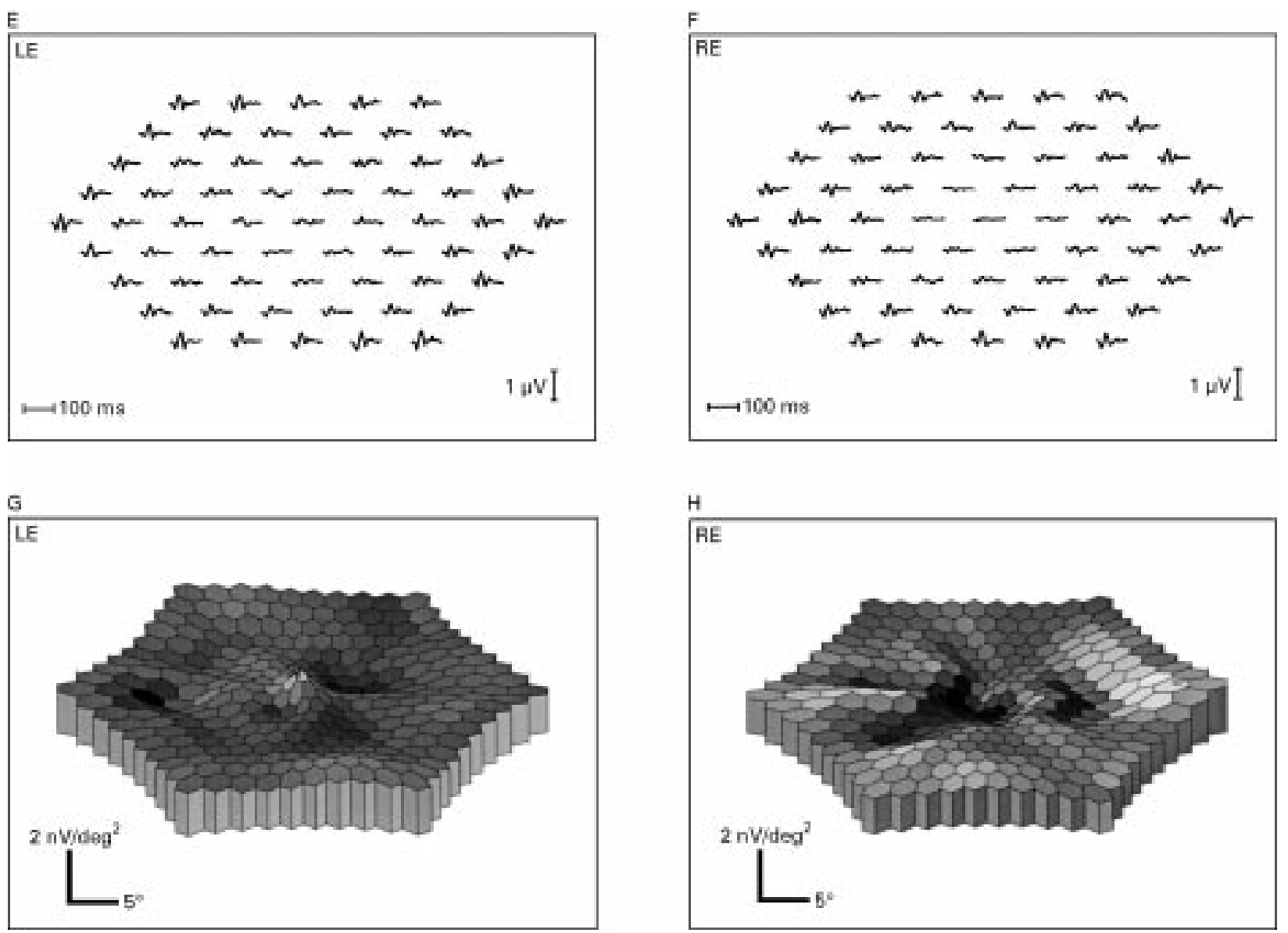

Figure 6 (continued) (E) Trace array of 61 focal ERGs (left eye). (F) Trace array of 61 focal ERGs (right eye). (G) Plot of response density (left eye). (H) Plot of response density (right eye).

Stargardt's macular dystrophy. ${ }^{33}$ Multifocal electroretinography may help to characterise the functional retinal topography in genetically defined subjects in the future.

Software and hardware were provided by Dr E Stutter, San Francisco, USA, and Tommy AG, Erlanger, Germany. We thank R Ehlers, J Isensee, and S Scholz for their technical assistance, Dr A Kurtenbach for commenting the manuscript, and K
Roller for graphical support. The work was supported by the Deutsche Forschungsgemeinschaft, Bonn, Germany (grants $\mathrm{Zr}$ 1/10-1; Zr 1/10-2; Ru 457/1-3).

1 Stargardt K. Über familäre, progressive Degeneration in der Maculagegend dens Auges. Graefes Arch Chin Exp Ophthalmol 1909;71:534-50.

2 Franceschetti A. Über tapeto-retinale Degenerationen mm Kindesalter. In: Souter H, ed. Entwicklung and Fortschritt in der Augenheilkunde. Stuttgart: F Enke, 1963:107-20.

3 Weleber RG. Stargardt's maculas dystrophy. Arch Ophthalmol 1994;112:752-4.

4 Fishman GA. Fundus flavimaculatus. A clinical classification. Arch Ophthalmol 1976;94:2061-7.

5 Noble KG, Carr RE. Stargardt's disease and fundus flavimaculatus. Arch Ophthalmol 1979;97:1281-5

6 Krill AE. Flecked retina diseases. In: Krill AE, Archer DB, eds. Hereditary retinal and choroidal diseases. Hagerstown: Harper and Row, 1977:739-824.

7 Fishman GA, Ferber M, Patel BS, Derlacki DJ. Visual acuity loss in patients with Stargardt's macular dystrophy. Ophthalmology 1987;94:809-14.

8 Lopez PF, Maumenee IH, de la Cruz Z, Green WR. Autosomal-dominant fundus flavimaculatus. Clinicopathologic correlation. Am f Ophthalmol 1990;97:798-809.

9 Klien BA, Krill AE. Fundus flavimaculatus. Clinical, functional and histopathologic observations. Am f Ophthalmol 1967;64:3-23.

10 McDonnell PJ, Kivlin JD, Maumenee IH, Green WR. Fundis flavimaculatus without maculopathy. A clinicopathologic study. Ophthalmology 1986;93:116-9.

11 Eagle RC, Lucien AC, Bernadino VB, Yanoff M. Retinal pigment epithelium abnormalities in fundus flavimaculatux. Ophthalmology 1980;87:1189-200.
12 Sitter EE, Tran D. The field topography of ERG components in man -I The photopic luminance response. Vision Res 1992;32:433-46.

13 Hood DC, Siple W, Holopigian K, Greenstein V. A comparison of the components of the multifocal and full-field ERGs. Wis Neurosci 1997;14:533-44.

14 Siple WH, Siegel IM, Carr RE, Mayon C. Evaluating macular function using the focal ERG. Invest Ophthalmol Dis Sci 1986;27:1123-30.

15 Sandburg MA, Jacobson SG, Benson EL. Fovea cone electroretinograms in retinitis pigmentosa and juvenile macular degeneration. Am f Ophthalmol 1979;88:702-7.

16 Miyake Y, Yanigida K, Kondo T, Yagasaki K, Ohta I. Subjective scotometry and recording of local electroretinogram and visual evoked response -system with television monitor of the fundus. $f_{p n} \mathfrak{f}$ Ophthalmol 1981;25:438-48.

17 Parks S, Keating D, Williamson TH, et al. Functional imageing of the retina using the multifocal electroretinograph: a control study. Br f Ophthalmol 1996;80:831-4.

18 Bears MA, Sitter EE. Imaging localized retinal dysfuncton with the multifocal electroretinogram. Opt Soc Am fA 1996;13:634-40.

19 Kongo M, Miyake Y, Horiguchi M, Suzuki S, Tanikawa A. Clinical evaluation of multifocal electroretinogram. Invest Ophthalmol Vic Sci 1995;36:2146-50.

20 Kretschmann U, Rüther K, Usui T, Zrenner E. ERG campimetry using a multi-input stimulation technique for mapping of retinal function in the central visual field. $O p h-$ thalmic Res 1996;28:303-11.

21 Marmor MF, Zrenner E. Standard for clinical electroretinography (1994 upgrade). Doc Ophthalmol 1995;89:199210.

22 Sitter EE. A deterministic approach to nonlinear systems analysis. In: Pinter RB, Nabet B, eds. Nonlinear vision: determination of neural receptive fields, function, and networks. Bock Ration: CRC Press, 1992:171-220.

23 Sitter EE. The fast $\mathrm{m}$-transform: a fast computation of cross-correlations with binary m-sequences. Siam f Comput 1991;20:686-94.

24 Curcio CA, Sloan KR, Katina RE, Hendrickson AE. Human photoreceptor topography. F Comp Neural 1990;

25 Sandberg MA, Effron MH, Berson EL. Focal cone electroretinograms in dominant retinitis pigmentosa with reduced penetrance. Invest Ophthalmol Wis Sci 1978;17: 1096-101.

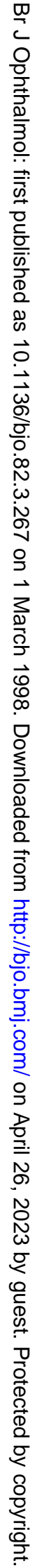


26 Fish GE, Birch DG. The focal electroretinogram in the clinical assessment of macular disease. Ophthalmology 1989;96:109-14.

27 Holder GE. Significance of abnormal pattern electroretin ography in anterior visual pathway dysfunction. $\mathrm{Br} \mathcal{F ~ O p h}$ thalmol 1987;71:166-71.

28 Seeliger MW, Kretschmann UH, Ruether KW, ApfelstedtSylla E, Zrenner E. ERG campimetry in retinitis pigmentosa. Invest Ophthalmol Vis Sci 1996;37:S341.

29 Hood DC, Holopigian K, Greenstein VC, et al. Do the delays in the cone ERG from patients with RP indicate global retinal damage? Invest Ophthalmol Vis Sci 1996;37: S341.

30 Kretschmann UH, Ruether KW, Zrenner E. Observations regarding the waveform of the ERG recorded with the m-sequence stimulation technique. Invest Ophthalmol Vis Sci 1996;37:S346.

31 Zrenner E. Flecked retina diseases. In: Cordella M, Baratta G, Macalusco C, eds. Retinite pigmentosa, movimenti oculari e ambliopia, glaucoma. Rome: Casa Editricie Mattioli, 1991: 393-7.

32 Kretschmann U, Usui T, Ruether K, Zrenner E. Electroretinographic campimetry in a patient with crystalline troretinographic campimetry in a patient with
retinopathy. Ger f Ophthalmol 1997;5:399-403.

33 Allikmets R, Singh N, Sun H, et al. A photoreceptor cell-specific ATP-binding transporter gene (ABCR) is mutated in recessive Stargardt macular dystrophy. Nat Genet 1997;15:236-46. 